\title{
Spectroscopy of a Single Si Donor by the Resonant Tunnelling Experiment
}

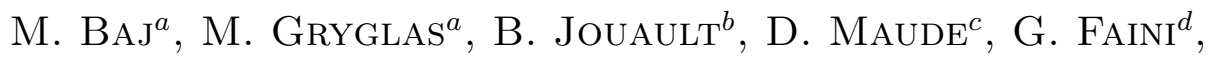 \\ U. GEnNSER ${ }^{d}$ AND A. CAVAnna ${ }^{d}$ \\ ${ }^{a}$ Institute of Experimental Physics, Warsaw University \\ Hoża 69, 00-681 Warsaw, Poland \\ ${ }^{b}$ Groupe d'Etude des Semiconducteurs, Université Montpellier II, CNRS \\ 34095 Montpellier, France \\ ${ }^{c}$ Grenoble High Magnetic Field Laboratory, CNRS \\ 25 Avenue des Martyrs, 38042 Grenoble, France \\ ${ }^{d}$ Groupe de Physique et Technologie des Nanostructures LPN - CNRS \\ 91460 Marcoussis, France
}

\begin{abstract}
Resonant tunnelling via single impurities placed in a single barrier was experimentally studied. The typically observed structures in the currentvoltage characteristics seem to be paired. Such a pair can be interpreted as ground and excited states of an impurity, related to $X_{x y}$ and $X_{z}$ valleys, which is in agreement with the results obtained for big mesas. However, it is not clear why $X_{x y}$ states can be seen without any phonon participation.
\end{abstract}

PACS numbers: 73.63.-b, 73.40.Gk, 73.20.Hb, 72.10.Di, 72.10.Fk

\section{Introduction}

The resonant tunnelling experiments in which single objects are involved proved to be an efficient tool to study the properties of the electron gas $[1,2]$. However, as the $I(V)$ curves are usually very complex for high bias, only the near-threshold structures are often analysed. In this paper, we present a set of data where several structures are clearly resolved in a relatively high bias range. The observed similarities of the structures suggest that there must be a common origin of these peaks.

\section{Structures studied}

The studied system was GaAs/AlAs/GaAs structure, grown by MBE on a [100] GaAs wafer $\left(n_{d}=1 \times 10^{18} \mathrm{~cm}^{-3}\right)$. The $10 \mathrm{~nm}$ AlAs layer was $\delta$-doped in 
the middle, with silicon having the planar concentration of $n_{\delta}=1 \times 10^{10} \mathrm{~cm}^{-2}$. The AlAs barrier was separated from the heavily doped GaAs contacts $\left(n_{d}=\right.$ $1 \times 10^{18} \mathrm{~cm}^{-3}$ ) by $200 \mathrm{~nm}$ GaAs undoped spacers in order to obtain high quality two-dimensional electron gas (2DEG). Mesa structures of different lateral sizes ranging from $500 \mu \mathrm{m}$ down to $100 \mathrm{~nm}$ were fabricated. This allowed us to have different numbers of donors within the junction, starting from more than $10^{6}$ for the largest mesas to less than 10 for the smallest ones.

\section{Experimental results}

Figure 1 shows the current-voltage characteristics for the $900 \mathrm{~nm}$ wide mesa, measured at $30 \mathrm{mK}$. For such a small mesa the number of impurities expected in the barrier is about 80 . As one can see, the $I(V)$ characteristics has diode-like behaviour, which is due to both resonant and non-resonant tunnelling. Above $0.7 \mathrm{~V}$ there is a series of fine structures resolved in the curve. The inset of Fig. 1

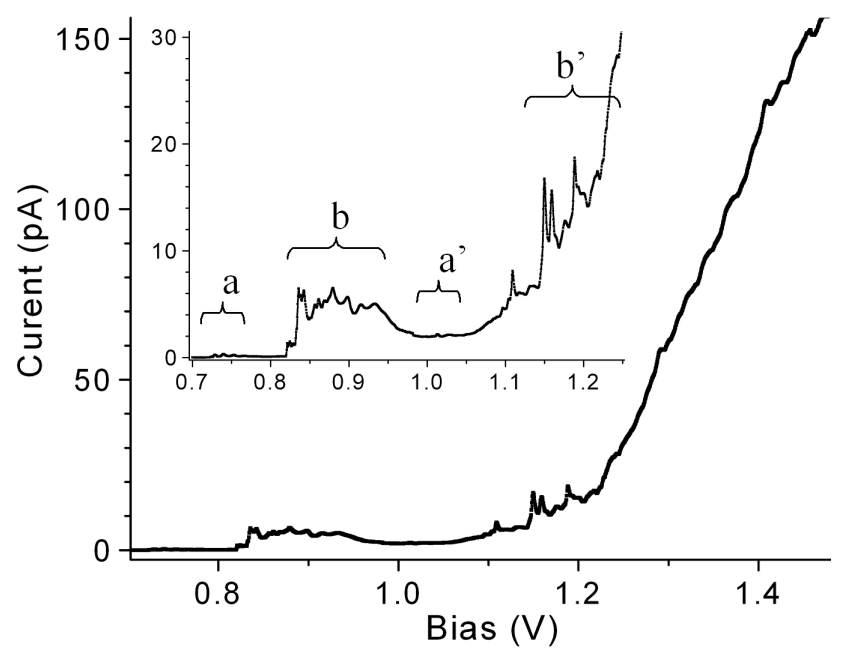

Fig. 1. $I(V)$ curve for $900 \mathrm{~nm}$ mesa measured at $30 \mathrm{mK}$. The interesting feature in the characteristics appears starting from $0.7 \mathrm{~V}$.

presents a magnification of the low bias region. The structure present at about $0.9 \mathrm{~V}$, marked with $b$, reflects the tunnelling via a single state of one impurity and it was discussed in more detail in [3]. Here we want to emphasize that the complicated shape of each structure (e.g. for $b$ - many sharp peaks of the current) reflects the local density of states in the emitter (2DEG in the GaAs region adjacent to the AlAs layer) in the neighbourhood of impurity, thus is characteristic of a given impurity. It is interesting to note that among all the structures we observe some which seem to be paired, e.g. $a$ with $a^{\prime}$ and $b$ with $b^{\prime}$ (Fig. 1). They are presented in Fig. 2 where the curves from higher bias (grey) are superimposed on the lower bias region (black) by a linear transformation of the voltage axis. There 

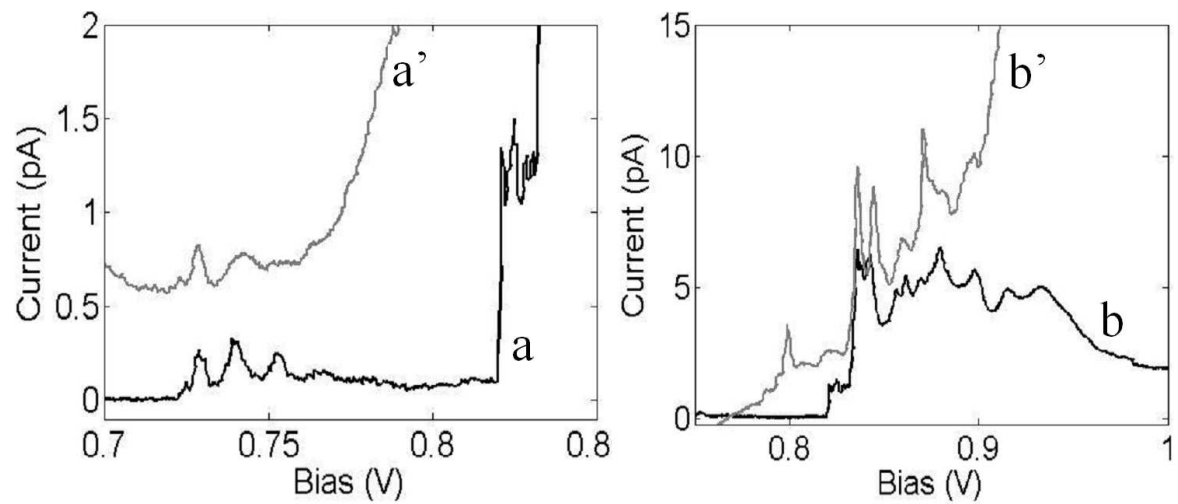

Fig. 2. The pairs of structures $a$ and $a^{\prime}$ and $b$ and $b^{\prime}$ (cf. Fig. 1) superimposed with a linear transformation of bias axis.

are evident similarities between both components of each pair, which suggests that most probably they originate from the same donor.

In order to find out what the possible origin of these structures is, the energy separation between them was estimated. We took advantage of the magnetic field studies of the Landau quantization for the structure $b$, reported in [3]. Knowing cyclotron energy $\hbar \omega_{\mathrm{c}}$ and separation between the Landau levels in the bias voltage scale, a so-called leverage factor $\alpha=\Delta U / \Delta E$ was found $(12.3 \mathrm{mV} / \mathrm{meV})$. As derived, the leverage factor was true for structure $b$, but could have also been calculated for the structure $b^{\prime}$, since the multiplicative factor needed to superimpose the two structures was known. To obtain the dependence of $\alpha$ on the bias voltage in a wider range, an extrapolation was made, taking into account also the structures $a$ and $a^{\prime}$ for which the above mentioned multiplicative factor was also

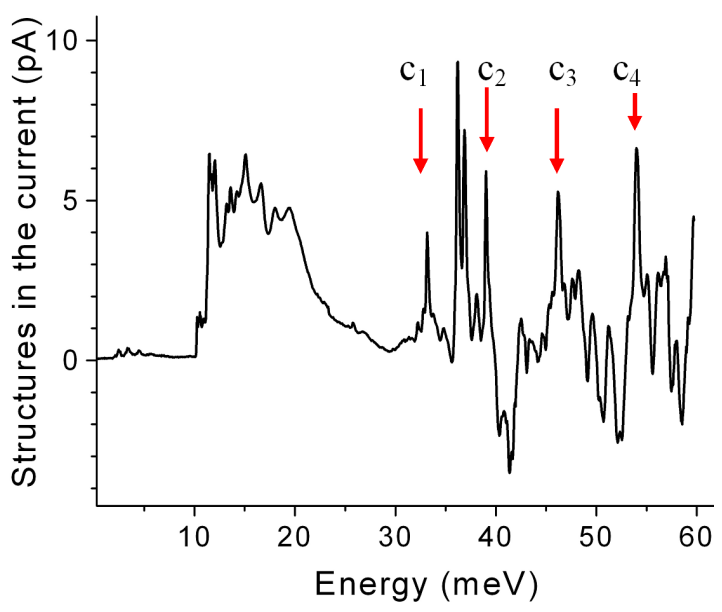

Fig. 3. The structures in the current after subtraction of the rising slope from Fig. 1 showed as a function of energy. The zero of energy is set arbitrarily. 
known (see Fig. 2). This procedure allowed to recalculate the bias axis into energy scale, and in particular, to obtain the separations between the structures:

- $a, a^{\prime}: 23 \mathrm{meV}$,

- $b, b^{\prime}: 25 \mathrm{meV}$,

where the accuracy of the estimation is of about $5 \mathrm{meV}$. Beside these two pairs, there are also many other structures visible in the $I(V)$ characteristics. They are presented in Fig. 3, which shows the current as a function of energy after subtraction of the rising background. In particular, four of them, marked $c_{1}$ to $c_{4}$ are similar in shape and almost equally separated $(6 \div 8 \mathrm{meV})$. Two of them, $c_{1}$ and $c_{2}$, have also been visible on the grey curve in Fig. 2 (right part), disturbing the agreement between structures $b$ and $b^{\prime}$.

\section{Discussion}

We start the discussion with the paired structures. The similarities between the constituents of the pairs mean that the local environment of the objects, which gives rise to those pairs, is identical. Therefore the structures observed at higher bias can be interpreted as coming from the excited states of the same impurity. Another possibility is that the paired structure is a tunnelling via the same state of the impurity with the participation of phonons.

Silicon donor in AlAs is a shallow impurity, related to $X$ minimum, which due to biaxial strain and anisotropic effective masses is split into two $X_{x y}$ and $X_{z}$ groups of valleys, which in principle can be mixed by the donor potential. However, symmetry arguments for donor in bulk AlAs lead to a conclusion that donor states can be treated as being related to independent valleys [4]. Besides, during the measurement the impurity is subjected to the electric field of several tens of $\mathrm{kV} / \mathrm{cm}$, which in turn influences the donor state [5]. Moreover, its energy depends on the donor position in the structure [6]. Basing on $[4,5]$ and $[6]$ one finds that for the Si impurity placed in the centre of the AlAs layer, its shallow-donor ground state is related to $X_{x y}$ minima, and the first excited state is related to $X_{z}$ minimum and lies $24 \mathrm{meV}$ above the ground one [7]. The higher excited states are separated from the $X_{z}$-related first excited one by a few meV.

Such predictions for donor spectrum are in a very good agreement with the separations within structures $a, a^{\prime}$ and $b, b^{\prime}$, reported in this paper. The presence of two resolved sets $a, a^{\prime}$ and $b, b^{\prime}$ indicates that there are at least two impurities in the junction, whose position varies and which show up at different bias. Similar doublets with approximately the same splitting have been observed for macroscopic mesas. Figure 4 shows the differential conductance $\sigma(V)$ measured at $4 \mathrm{~K}$ for the undoped sample (dashed line) and also for the doped one (solid one), with a few millions of donors involved in the tunnelling. The hill at about $1 \mathrm{~V}$, present only for the doped structure, is composed of two constituents. The separation between them is about $0.3 \mathrm{~V}(\approx 20 \mathrm{meV})$, which nicely agrees with the prediction for energy 


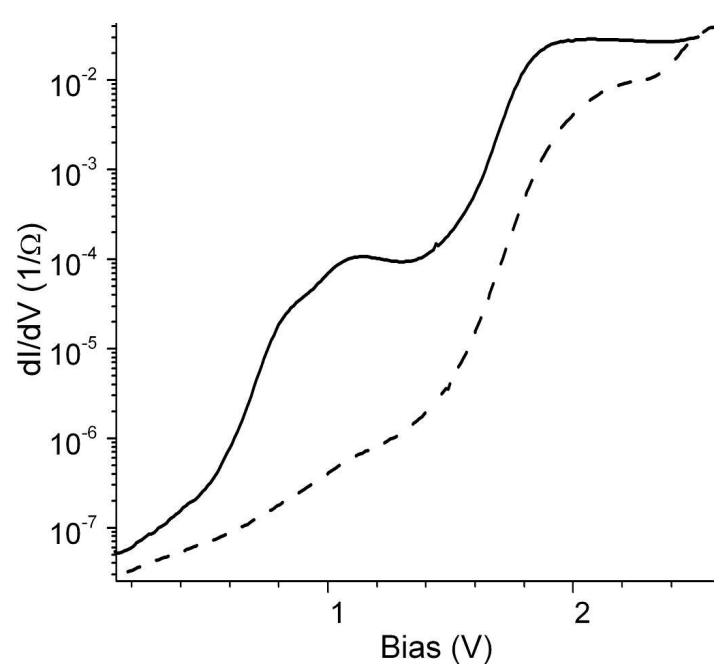

Fig. 4. Differential conductance versus bias applied to the diode for $500 \times 500 \mu \mathrm{m}$ mesa for two cases: the donors introduced in the barrier (solid line) and no intentional donors (dotted line).

difference between the ground and excited states of the donor. Thus, the doublet observed in Fig. 4 is ascribed to the resonant tunnelling via donors related to $X_{x y}$ (lower bias) and $X_{z}$ (higher bias) minima [8].

One point in this reasoning is delicate and is not addressed in the literature: the two donor states significantly differ in their in-plane momentum - it is substantial for the state related to $X_{x y}$ valley and small for $X_{z}$ valley one. Thus, one could expect that for electron from GaAs (having small in-plane momentum since it is related to $\Gamma$ minimum) a momentum mismatch in tunnelling via $X_{x y}$-related state can drastically reduce the probability of the tunnelling and in consequence, contribution of $X_{x y}$-related states to tunnel current. The transition via $X_{x y}$ states calls for the participation of phonons that would supply the missing in-plane momentum. Such a rule was observed in the experimental investigations of the resonant tunnelling via AlAs quantum well states [9]. The transitions involving states having high in-plane momenta were observed only with transversal acoustic (AlAs) phonons from the Brillouin zone edge, whereas states with low in-plane momenta were observed directly.

It is not evident that such reasoning should be applied to the tunnelling via localised levels. Our experimental data cannot be easily explained by phonon participation. Indeed, if the tunnelling through $X_{x y}$ state was not possible without phonons, then the first (non-prime) structure must be due to the tunnelling via $X_{z}$-related donor state, while the second should be related to phonon-assisted tunnelling via $X_{x y}$ state. Taking into account the phonon energies in AlAs or GaAs (from $X$ point) and the separation between the ground and excited state of the donor, the expected separation within each pair should be of about $5 \mathrm{meV}$, 
while experimentally it is about $24 \mathrm{meV}$. Besides, there is no reason to have only one phonon replica and so sets of similar structures should be seen - whereas for big mesas only doublet structure is clearly resolved.

One can think of physical reasons why the mismatch of the in-plane momentum does not have to block the resonant tunnelling through the localised states. A perturbing potential introduced by the donor may lead to mixing of $\Gamma(\mathrm{GaAs})$ and $X(\mathrm{AlAs})$ states, making tunnelling possible. Moreover, the disorder at the interface in the plane of the layer, with a characteristic period of the order of a lattice constant, can contribute to the relaxation of the in-plane momentum.

Thus, most plausible origin of the doublet structures $\left(a, a^{\prime}\right.$ and $\left.b, b^{\prime}\right)$ is the tunnelling via ground and first excited states of two independent impurities. The origin of the regular structures $c_{1} \div c_{4}$ observed at higher energies is not clear. The similar separations between them suggest that they could be multi-phonon replicas. However, the value of the separation is too small to fit any peak of $X$-point phonon density of states in GaAs or AlAs.

\section{References}

[1] P.C. Main, A.S.G. Thornton, R.J.A. Hill, S.T. Stoddart, T. Ihn, L. Eaves, K.A. Benedict, M. Henini, Phys. Rev. Lett. 84, 729 (2000); E. Lind, B. Gustafson, I. Pietzonka, L.-E. Wernersson, Phys. Rev. B 68, 033312-1 (2003).

[2] M. Gryglas, M. Baj, B. Chenaud, B. Jouault, A. Cavanna, G. Faini, Phys. Rev. B 69, 165302 (2004).

[3] B. Jouault, M. Gryglas, G. Faini, U. Gennser, A. Cavanna, M. Baj, D.K. Maude, Phys. Rev. B 73, 155415 (2006).

[4] T.N. Morgan, Phys. Rev. B 34, 2664 (1986); Phys. Rev. Lett. 21, 819 (1968).

[5] J.A. Blum, C. Priester, G. Allan, Phys. Rev. B 32, 2378 (1985).

[6] G. Weber, Appl. Phys. Lett. 67, 1447 (1995); G.N. Carneiro, G. Weber, Phys. Rev. B 58, 7829 (1998).

[7] M. Gryglas, Ph.D. thesis, Warsaw University, Warsaw 2004, p. 33.

[8] I.E. Itskevich, L. Eaves, P.C. Main, M. Henini, G. Hill, Phys. Rev. B 57, 7214 (1998).

[9] J.J. Finley, R.J. Teisser, M.S. Skolnick, J.W. Cockburn, G.A. Roberts, R. Grey, G. Hill, M.A. Pate, R. Planel, Phys. Rev. B 58, 10691 (1998). 